HNO 2021 $69: 907-912$

https://doi.org/10.1007/s00106-020-00989-9

Angenommen: 21. Oktober 2020

Online publiziert: 13. Januar 2021

๑) Der/die Autor(en) 2021

J. Künzel $\left.\right|^{1}$ A. Bozzato ${ }^{2}$ B. P. Ernst ${ }^{3} \cdot$ T. Fuhrmann ${ }^{4}$ I. Ugele E C. Scherl $^{5}$. M. Schapher ${ }^{6} \cdot$ G. F. Volk $\cdot$ N. Mansour ${ }^{8} \cdot$ A. Knopf ${ }^{3} \cdot$ C. Bohr ${ }^{1} \cdot$ K.-F. Hamann ${ }^{9}$ ${ }^{1}$ Klinik und Poliklinik für Hals-Nasen-Ohren-Heilkunde, Universitätsklinikum Regensburg, Regensburg, Deutschland

${ }^{2}$ Klinik und Poliklinik für Hals-, Nasen-, Ohrenheilkunde, Universitätsklinikum des Saarlandes, Homburg, Deutschland

${ }^{3}$ Hals-, Nasen-, Ohrenklinik und Poliklinik, Universitätsmedizin Mainz, Mainz, Deutschland

${ }^{4}$ Fachbereich Ingenieur- und Naturwissenschaften, Hochschule Merseburg, Merseburg, Deutschland

${ }^{5}$ Klinik für Hals-Nasen-Ohren-Heilkunde, Kopf- und Halschirurgie, Universitätsmedizin Mannheim, Mannheim, Deutschland

${ }^{6}$ Hals-Nasen-Ohren-Klinik, Kopf- und Halschirurgie, Universitätsklinikum Erlangen, Erlangen, Deutschland ${ }^{7}$ Klinik für Hals-, Nasen- und Ohrenheilkunde, Universitätsklinikum Jena, Jena, Deutschland

${ }^{8}$ Klinik für Hals-, Nasen- und Ohrenheilkunde, Universitätsklinikum Freiburg, Freiburg, Deutschland

${ }^{9}$ HNO-Klinik Dr. Gaertner, München, Deutschland

\title{
Qualität in der Befundung von Kopf- und Halssonographien an Universitätskliniken - eine Stichprobe
}

\section{Hintergrund und Fragestellung}

Die Ultraschalldiagnostik genießt eine weite Verbreitung und gilt für den Radiologen, Hals-Nasen-Ohren-Arzt oder Mund-Kiefer-Gesichts-Chirurgen als Standard in der primären Abklärung diverser Pathologien. Insbesondere in der Tumornachsorge hat die Ultraschalldiagnostik ihren besonderen Stellenwert. Mitte der 1980er-Jahre wurde die Sektion „Kopf-Hals“ der Deutschen Gesellschaft für Ultraschall in der Medizin (DEGUM) gegründet. Mitte der 1990erJahre wurde die Ultraschalldiagnostik im Kopf-Hals-Bereich dann fester Bestandteil der Assistentenausbildung. In der HNO-Facharzt-Weiterbildung werden über 200 Ultraschalluntersuchungen des Kopf-Hals-Bereichs mit entsprechender Dokumentation gefordert. Zusätzlich werden aktuelle Ausbildungsstandards von der DEGUM vorgeschlagen (https:// www.degum.de), welche neben separat belegbaren Kursen auf Basis des DEGUM-Ausbildungscurriculums die Zertifizierung über einzelne Kompetenzstufen anbietet. Im Jahr 2018 pu- blizierten Heyduck et al. die Ergebnisse einer deutschlandweiten Umfrage zur Wertigkeit der Ultraschalldiagnostik im Kopf- und Halsbereich [9]. Vor allem die Weichteil- und onkologische Diagnostik hatten sowohl bei Kollegen in der Niederlassung als auch in den Kliniken einen hohen Stellenwert. Die Auswertung ergab, dass niedergelassene Kollegen im Vergleich zu den Kliniken bereits 2018 einen höheren Grad der Digitalisierung erreichten. Es erfolgte bereits zu diesem Zeitpunkt zu 55\% eine komplett digitale Dokumentation, hingegen bei $70 \%$ der Befragten in der Klinik weiterhin eine manuelle Dokumentation mit Ausdruck der Befunde. Die digitale Dokumentation erscheint wichtig, um die Qualität der sonographischen Dokumentationen $\mathrm{zu}$ verbessern und $\mathrm{zu}$ standardisieren, denn nur so sind Befunde in angemessener Qualität archivierbar, abrufbar und können beispielsweise im Rahmen von Tumorkonferenzen sinnvoll zur Entscheidungsfindung hinzugezogen werden. Die moderne Informationstechnologie (KIS, PACS) und die elektronische Patientenakte setzen die digitale Inte- gration von Bild- und Befunddaten in medizinische Informationssysteme voraus. Die Einbindung von Befunden anderer bildgebender Verfahren wie Computertomographie (CT) und Magnetresonanztomographie (MRT) sind hier als Maßstab zu betrachten [9]. Zuletzt häuften sich Publikationen, welche die Implementierung standardisierter Befunddokumentation ebenfalls für die Kopf- und Halssonographie fordern [1, 3 , 5, 10, 17-19]. Ernst et al. zeigten, dass die strukturierte digitale Befunderhebung im Vergleich zur handschriftlichen in puncto Vollständigkeit, Lesbarkeit und Zeiteffizienz nach einer entsprechenden Implementierungsphase überlegen ist [5]. Die vorliegende Arbeit ermittelt stichprobenartig die Qualität von routinemäßig angefertigten Kopf- und Halssonographiebefunden nach Kriterien der KVB an einer Auswahl deutscher HNO-Universitätskliniken. 
Tab. 1 Art der Bild-und Befunddokumentation in den einzelnen Kliniken

\begin{tabular}{l|llllll} 
Standort & Befund & Bild & Struktur & Textbausteine & $\begin{array}{l}\text { Befund und Bild Kombinati- } \\
\text { on }\end{array}$ \\
\hline Erlangen & Digital & Digital & Ja & Ja & KIS/PACS integriert \\
\hline Freiburg & Papier & Thermoprint & Nein & Nein & Tacker \\
Homburg & Digital & Digital & Ja & Ja & KIS/PACS integriert \\
\hline Jena & Digital & Digital & Ja & Ja & KIS/PACS separiert \\
Mainz & Digital & Digital & Nein & Nein & KIS/PACS separiert \\
Mannheim & Papier & Thermoprint & Nein & Nein & Tacker \\
Regensburg & Papier & Thermoprint & Nein & Nein & Tacker \\
\hline
\end{tabular}

\section{Studiendesign und Unter- suchungsmethoden}

Insgesamt wurden 70 zufällig ausgewählte, anonymisierte schriftliche Befunde einschließlich des zugehörigen Bildmaterials von insgesamt 7 HNO-Universitätskliniken (Erlangen, Freiburg, Homburg, Jena, Mainz, Mannheim, Regensburg) nach KV-Kriterien durch einen erfahrenen Prüfer der KV Bayern (KFH) retrospektiv ausgewertet. Die Anfrage zur Studienteilnahme der Zentren erfolgte nicht systematisch, sondern selektiv vorwiegend auf der Basis einer Sitzung der Kopf- und Halssektion der DEGUM. Jede der teilnehmenden Kliniken stellte 10 Befunde aus der allgemeinen Klinikroutine zur Auswertung bereit. Allen teilnehmenden Kliniken stehen hochmoderne Ultraschallgeräte zur Verfügung. Die HNO-Universitätskliniken Homburg und Freiburg sind DEGUMzertifizierte Zentren und verfügen über DEGUM-Kursleiter der Stufe III. Erlangen, Mainz und Regensburg verfügen über Mitarbeiter mit der DEGUM-Stufe III und Jena über Stufe-IIb-Kursleiter. In Erlangen, Freiburg und Mainz wird ein DEGUM-zertifizierter Ausbildungskurs der Kopf- und Halssonographie angeboten. Darüber hinaus beschäftigen alle diese Kliniken weitere Mitarbeiter mit DEGUM-Stufenzertifizierung.

Folgende 16 Parameter wurden für die Qualitätsbewertung der Dokumentation zugrunde gelegt: Fragestellung nicht erkennbar; Schnittebenen nicht nachvollziehbar; anatomische Bezeichnungen falsch/fehlen; pathologischer Befund nicht markiert; zweite Ebene fehlt; Messmarker fehlen/unvollständig;
Messwerte unvollständig/fehlen; Befund kann nicht belegt werden; Bilddokumentation überstrahlt; Bilddokumentation unterstrahlt; Seitenbezeichnung fehlt; Kontrast nicht ausreichend; Eindringtiefe unzureichend; Befundbeschreibung lückenhaft; Diagnose fehlt/falsch; therapeutische/diagnostische Konsequenz fehlt.

In der Ultraschallvereinbarung heißt es in $\$ 10 \mathrm{im}$ Wortlaut, dass „bei pathologischem Befund eine Darstellung in 2 Schnittebenen oder - wenn dies nicht möglich ist - in einer Schnittebene (nur bei B-Modus)“mit „zur Befunderstellung notwendigen Messwerten und Messmarkern" gefordert wird. Im Rahmen der vorliegenden Auswertung wurden für eine korrekte Befundung in jeder Bildebene 2 Messmarker bzw. 2 Messwerte vorausgesetzt. Sofern also beispielsweise ein Befund nicht in 2 Ebenen markiert ist und/ oder eine der Ebenen nicht in 2 Orientierungen markiert wurde, sind die Messmarker fehlerhaft oder unvollständig und folglich auch die Messwerte unvollständig oder fehlend. In der Diskussion dieser Arbeit wird dieser Sachverhalt vertiefend erörtert.

Die Datenauswertung erfolgte deskriptiv. Auf eine exakte Darstellung der Einzelergebnisse nach Klinik wurde aus Diskretionsgründen verzichtet. Aufgrund des rein retrospektiven Charakters der Studie ohne Patientenbezug wurde kein Ethikvotum beantragt. Die Studie erfolgte unter Einhaltung der Vorgaben der aktuellen Fassung der Deklaration von Helsinki.
Tab. 2 Fehlerhäufigkeit in \% nach Befund-

parametern

Fragestellung nicht erkennbar

15,9

Schnittebenen nicht nachvollziehbar

20,3

Anatomische Bezeichnungen falsch/

fehlen

Pathologischer Befund nicht markiert

Zweite Ebene fehlt

Messmarker fehlen/unvollständig

Messwerte fehlen/unvollständig

Befund kann nicht belegt werden

Bilddokumentation überstrahlt

Bilddokumentation unterstrahlt

4,3

Seitenbezeichnung fehlt

2,9

Kontrast nicht ausreichend

Eindringtiefe unzureichend

Befundbeschreibung lückenhaft

Diagnose fehlt/falsch

Therapeutische/diagnostische Konsequenz fehlt

\section{Ergebnisse}

Die Art der Bild- und Befunddokumentation sowie die Archivierungsmethode in den einzelnen Kliniken ist in • Tab. 1 dargestellt.

Von den 70 Befunden konnten 69 in die vorliegende Studie eingeschlossen und ausgewertet werden. Lediglich ein Befund musste aufgrund schlechter Druckqualität der Befundkopie von der Auswertung ausgeschlossen werden. Die Dokumentationsvollständigkeit der gesamten Stichprobe variierte von $50-100 \%$ mit einem Mittelwert von $80,6 \%$ (Standardabweichung 14,5\%). Insgesamt waren 9 Befunde vollständig und korrekt dokumentiert (13\%).

Die Dokumentationsvollständigkeit der einzelnen Kliniken lag aufsteigend sortiert bei $68,1 \%, 73,2 \%, 79,4 \%, 79,4 \%$, $81,9 \%, 90,7 \%$ und $93 \%$.

Die Häufigkeiten der Mängel in Bezug auf die einzelnen ausgewerteten Befundparameter ist in $\bullet$ Tab. 2 dargestellt.

Es zeigt sich, dass mit einer Häufigkeit von über $25 \%$ besonders die Befundbestandteile „therapeutische/diagnostische Konsequenz", „Messmarker und Messwerte“ und „anatomische Bezeichnungen“ fehlerhaft bzw. unvollständig waren. In - Abb. 1a-d sind exemplarisch derartige Befunde veranschaulicht. 
HNO 2021 · 69:907-912 https://doi.org/10.1007/s00106-020-00989-9

(c) Der/die Autor(en) 2021

\section{J. Künzel · A. Bozzato · B. P. Ernst · T. Fuhrmann · I. Ugele · C. Scherl · M. Schapher · G. F. Volk · N. Mansour · A. Knopf · C. Bohr · K.-F. Hamann} Qualität in der Befundung von Kopf- und Halssonographien an Universitätskliniken - eine Stichprobe

\section{Zusammenfassung}

Hintergrund. Die Ultraschalldiagnostik gilt für den Radiologen, Hals-Nasen-OhrenArzt (HNO) oder Mund-Kiefer-GesichtsChirurgen als Standard in der Abklärung zahlreicher Pathologien. Es besteht ein Konsens, dass die digitale Dokumentation heute dringend notwendig ist, um die Qualität der sonographischen Dokumentationen zu verbessern und zu standardisieren. Es häufen sich Publikationen zur Implementierung standardisierter Befunddokumentation einschließlich der Kopf- und Halssonographie. Ziel der Arbeit. Die vorliegende Arbeit zielt darauf ab, die Qualität von routinemäßig angefertigten Kopf- und Halssonographiebefunden nach Kriterien der Kassenärztlichen Vereinigung (KV) Bayern an einer Auswahl deutscher HNO-Universitätskliniken stichprobenartig zu ermitteln.

Material und Methoden. Insgesamt wurden retrospektiv 70 zufällig ausgewählte, anonymisierte schriftliche Befunde einschließlich Bildmaterial von insgesamt 7 HNO-

Universitätskliniken stichprobenartig nach KVKriterien durch einen erfahrenen Prüfer der KV Bayern ausgewertet und deskriptiv analysiert. Ergebnisse. Von 70 Befunden konnten 69 ausgewertet werden. Die Dokumentationsvollständigkeit lag im Mittel bei 80,6\%. Neun Befunde waren vollständig korrekt dokumentiert (13\%). Die Dokumentationsvollständigkeit der einzelnen Kliniken lag zwischen $68,1 \%$ und $93 \%$. Mit $88,5 \%$ vs. $75 \%$ erbrachte eine strukturierte Befundung eine höhere Befundvollständigkeit. In $75 \%$ der Fälle verfügten die Kliniken mit strukturiertem Befund auch über digitale Dokumentationslösungen.

Schlussfolgerung. Die Vollständigkeit und Qualität von routinemäßig angefertigten Kopf- und Halssonographiebefunden an einer Auswahl von HNO-Universitätskliniken ist insgesamt optimierbar. Die Implementierung strukturierter Befundmasken und die Umstellung der analogen Dokumentation auf digitale Lösungen sowie Vernetzung mit dem Klinikinformationssystem (KIS) und Bildarchivierungs- und Kommunikationssystem (PACS) sollte weiter vorangetrieben werden. Darüber hinaus sind leitende Ärzte dazu angehalten, die Befundqualität unerfahrener Kollegen regelmäßig zu prüfen und im Rahmen der Facharztausbildung auf die Erfüllung entsprechender Standards wie der KV-Ultraschallvereinbarung hinzuarbeiten.

\section{Schlüsselwörter}

Digitale Befunddokumentation · Ultraschallausbildung · Strukturierte Befundung . Befundqualität · Befundvollständigkeit

\section{Quality in the appraisal of head and neck sonography results in university hospitals—a random sample}

\section{Abstract}

Background. Ultrasound diagnostics are widely used and are standard for radiologists, otolaryngologists, and oral and maxillofacial surgeons in the diagnostic work-up of various pathologies. There is agreement that digital documentation is urgently needed at present to improve and standardize the quality of sonographic documentation. There are more and more publications on the implementation of standardized documentation of findings in imaging diagnostics, including head and neck sonography.

Objective. The present work aims to determine the quality of routine head and neck sonography findings on a random basis, according to the criteria of the Bavarian Association of Statutory Health Insurance Physicians (KVB) at a selection of German university otolaryngology departments (ENT).
Materials and methods. A total of 70 randomly selected anonymized written findings including image documentation from seven ENT departments were retrospectively analyzed by an experienced KVB examiner concerning fulfilment of KVB criteria. The data were evaluated descriptively.

Results. Of the 70 reports, 69 were eligible for evaluation. The average documentation completeness was $80.6 \%$. A total of 9 findings were correctly documented in full (13\%). The documentation completeness of the individual departments was sorted in ascending order from $68.1 \%$ to $93 \%$. With $88.5 \%$ vs. $75 \%$, the hospitals with a structured report showed a higher level of completeness. In $75 \%$ of the cases the hospitals with structured reports also had digital solutions for reporting and image archiving.
Conclusion. In general, there is potential for optimization regarding the completeness and quality of routinely prepared head and neck sonography findings at the selected university ENT departments. The implementation of structured reporting masks and the conversion of analogue documentation into digital solutions as well as digital networking with the hospital information systems, picture archiving and communication systems should be promoted. Supervision by senior doctors is required to ensure the quality of findings of inexperienced colleagues and to help to achieve standards in reporting.

\section{Keywords}

Digital documentation of findings - Ultrasound training - Structured reporting - Quality of findings - Completeness of findings
Unter Berücksichtigung der Verwendung eines strukturierten Befundes und Textbausteinen ergeben sich für die Dokumentationsvollständigkeit unterschiedliche Mittelwerte. Mit $88,5 \%$ vs. $75 \%$ zeigten die Kliniken mit strukturierter Erhebung eine höhere Be- fundvollständigkeit. In $75 \%$ der Fälle verfügten die Kliniken mit strukturiertem Dokumentationsprozess ebenfalls über digitale Lösungen zur Befundung und Bildarchivierung.

\section{Diskussion}

Die Ultraschalldiagnostik im Kopf- und Halsbereich ist seit Jahrzehnten eine fest etablierte Untersuchungsmethode für eine Vielfalt an Kopf- und Halspathologien. Gerade zur Beurteilung von 

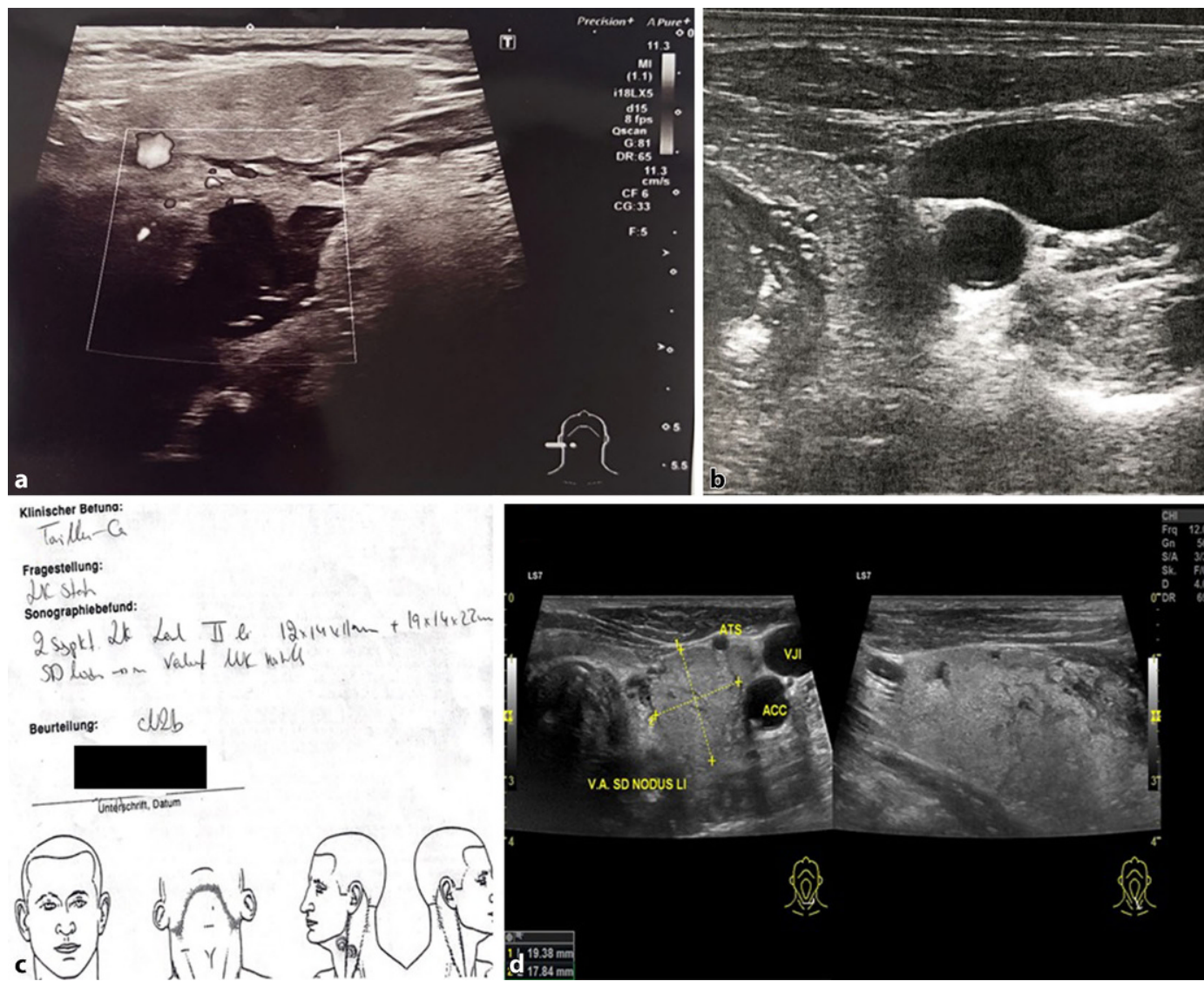

Abb. $1<$ a Darstellung einer Tonsillenloge der rechten Seite mit fehlenden Messmarkern und Messwerten sowie fehlenden anatonischen Bezeichnungen; b Bilddokumentation zervikal links ohne Piktogramm und fehlende anatomische Bezeichnungen; cschriftlicher Befund ohne Hinweis auf die diagnostische/therapeutische Konsequenz des auffälligen Halslymphknotenstatus; d Schilddrüsenknoten links mit fehlenden Messmarkern und Messwerten in der zweiten Ebene

Hals- oder Speicheldrüsenraumforderungen, beim Primär- oder Restaging von Kopf- und Halsmalignomen oder in der Tumornachsorge müssen hohe Anforderungen an die Befunddokumentation und Archivierung gestellt werden $[2,11,12,16]$. Die exakte topographische Zuordnung der Pathologie im Ultraschall ergibt sich für den nachbehandelnden Arzt erst komplementär aus der Bildbetrachtung mit dem zugehörigen schriftlichen Befund, denn im Gegensatz zur sonstigen klassischen Schnittbildgebung kann hier durch einfaches „Bildscrollen“ primär kein räumlicher Eindruck vermittelt werden. Die modernen Ultraschallgeräte bieten zwar weitgehend die Möglichkeit zur Videodokumentation, doch diese eigentlich optimale Dokumentationsvariante ist wegen häufig noch fehlender Schnittstellenanbindung an die PACS-Systeme und teilweise limitierten Speicherkapazitäten für Videos bisher kaum in der Praxis angekommen. Die fehlende Implementierung der Ultraschallbefunde in das KIS/PACS verhindert eine Demonstration von Ultra- schallbefunden und -videos im Rahmen von interdisziplinären Boards (Röntgendemonstration, Tumorkonferenz). Dies verringert aus rein technischen Gründen die Wertigkeit bzw. die Bedeutung der sonographischen Diagnostik gerade im interdisziplinären Diskurs im Vergleich zu den klassischen Schnittbildverfahren. Die Möglichkeit zur Korrelation von Ultraschallbefunden mit CT-/MRTAufnahmen ist häufig nicht in gleicher Qualität möglich. Da die Sonographie eine höhere Auflösung und Genauigkeit in der Lymphknotenbeurteilung als konventionelle Schnittbildverfahren aufweist, könnte die angesprochene Implementierung von Ultraschallbefunden somit zur weiteren Optimierung der Tumorboardentscheidungen beitragen [12].

Eine wesentliche Limitation der Studie ist, dass diese nur stichprobenartig die Art und die Qualität der Befunddokumentation in einer selektionierten Gruppe von Universitätsklinika abbildet. Rückschlüsse auf die Dokumentationsqualität in anderen universitären Häu- sern oder nichtuniversitären $\mathrm{HNO}-\mathrm{Ab}$ teilungen lassen unsere Daten nicht zu. Auch ohne statistische Überprüfung bzw. ohne Kontrollgruppe zeigen die vorliegenden Daten, dass selbst in einem selektionierten Kollektiv aus vorwiegend HNO-Universitätskliniken mit objektiv hoher Ultraschallexpertise gut $80 \%$ der Befunde nach den Kriterien der KV Bayern beanstandet wurden [13]. Lediglich $13 \%$ der Befunde gingen vollständig mit der hier angewendeten Auslegung der $\mathrm{KV}$-Vereinbarung konform.

Allerdings ist davon auszugehen, dass in Bezug auf "Messmarker/Messwerte“ die Ergebnisse besser ausgefallen wären, wenn die klinisch übliche Messmarkierung in 2 Ebenen mit 3 Orientierungen als unkritisch korrekt klassifiziert worden wäre. Letztlich wird hierbei allerdings angenommen, dass eine unregelmäßig gewachsene Raumforderung (z. B. polyzyklisches pleomorphes Adenom oder extranodal infiltrierende Lymphknotenmetastase) in 2 Ebenen mit 3 Orientierungen immer exakt erfasst wird. Die Vermessung einer Raumforderung in 2 um 
$90^{\circ}$ gedrehten Ebenen ergibt nur dann für die kurze Achse jeweils den gleichen Wert, wenn der Schallkopf exakt auf einem Punkt gedreht wird und dort die dritte Achse vermessen wird. Häufig werden in der Realität bewusst oder unbewusst die jeweiligen Maximaldurchmesser vermessen, die nicht unbedingt im $90^{\circ}$-Winkel zueinander stehen. In diesen Fällen generiert die zweite Orientierung in der zweiten Ebene mutmaßlich eine zusätzliche Information, die nicht a priori vernachlässigt werden sollte.

Ein benigner Lymphknoten zeichnet sich im Ultraschall durch eine glatt begrenzte, ovaläre Form mit einem Lymphknotendurchmesser von $<10 \mathrm{~mm}$ (kurze Achse) aus [7]. Ein weiteres Kriterium zur Differenzierung zwischen „benigne“ und "maligne“ ist mit dem Index der langen zur kurzen Achse des Lymphknotens beschrieben („Solbiati-Index“). Nach Steinkamp et al. lag die Sensitivität bei $95 \%$ bei einem „Cut-off-Wert“ des Index von 2 [20]. Folglich gilt in der Routine als Faustregel: Je runder der Lymphknoten ist, desto wahrscheinlicher liegt ein maligner Befund vor (Solbiati-Index $<2$ ). Die Lymphknotenform sollte jedoch nicht allein als Differenzierungskriterium verwendet werden, da benigne submandibuläre, im Mundboden (Level IA) gelegene, und intraparotideale Lymphknoten häufig eine rundliche Form haben [22]. Für die Parameter „kurze Achse“ und „Solbiati-Index“ ist die Darstellung in einer zweiten Ebene sogar gänzlich entbehrlich. Die Darstellung einer Raumforderung in 2 Ebenen dient allerdings insgesamt der besseren Reproduzierbarkeit. Es besteht wenig Evidenz für die Vermessung einer Raumforderung in 3 Orientierungen. Eine Volumenmessung ähnlich der Volumetrie der Schilddrüse ist lediglich eine mathematische Annäherung unter der Annahme eines ellipsoiden Körpers, was letztlich nicht generell für zervikale Raumforderungen präjudiziert werden kann. Sofern sinnvollerweise Maximaldurchmesser gemessen werden, ist nicht von einer exakt um $90^{\circ}$ verdrehten Messachse auszugehen und somit auch eine Vermessung der zweiten Ebene in 2 Orientierungen $\mathrm{zu}$ fordern. Noch genauer wäre formal eine Mehrfachvermessung einer Raumforde- rung mit Minimal- und Maximaldurchmessern in 2 Ebenen und 4 Orientierungen.

Zusammenfassend zeigen die Ergebnisse, dass in etwa $50 \%$ der Befunde Messmarker und Messwerte nicht korrekt gesetzt wurden, wobei entweder eine zweite Ebene gänzlich fehlte (ca. 20\%) oder die zweite Ebene gar nicht oder nur in einer Orientierung vermessen wurde (• Abb. 1d). Im Rahmen einer klinischen Studie sollten die im B-Scan verfügbaren Messparameter zervikaler Raumforderungen (z.B. kurze Achse, Solbiati-Index, Maximaldurchmesser, Min.-Max.-Durchmesser, 90 ${ }^{\circ}$ gedrehte zweite Ebene) weiter evaluiert und letztlich validiert werden. Die gängige Praxis der Vermessung einer Raumforderung in 2 Ebenen mit 3 mehr oder weniger zufällig ausgewählten Orientierungen sollte jedenfalls kritisch hinterfragt werden. Für die klinische Routine mit zeiteffektiver Befunderstellung sollte die Vermessung von Raumforderungen sinnvoll und gut reproduzierbar, gleichzeitig aber auch möglichst wenig fehleranfällig sein.

Sämtliche Befundparameter können in einer strukturierten digitalen Befundmaske vollständig vorgegeben, mittels Textbausteinen bzw. Drop-downMenüs befüllt und gerade häufig beanstandete Befundfelder „therapeutische/ diagnostische Konsequenz“, „Messmarker und Messwerte “ als Pflichtfelder abgefragt werden. Anatomische Bezeichnungen können zwar an den Ultraschallgeräten vorbelegt werden, sind dann jedoch aktiv vom Untersucher einzupflegen.

Die strukturierte Dokumentation hat sich als vielversprechender Ansatz zur Standardisierung des Befundes und zur Verbesserung der Gesamtberichtqualität verschiedener Diagnosemodalitäten, einschließlich Kopf- und Halsultraschall, erwiesen [3, 6, 14, 19,21]. Darüber hinaus bevorzugen überweisende und untersuchende Kollegen im Allgemeinen strukturierte Berichte gegenüber Freitextbefunden $[1,8,15]$. Da der Ultraschall ein Schlüsselelement bei der ambulanten Tumornachsorge und Operationsplanung ist, sind umfassende und verständliche Berichte unabdingbar. Der Befund jeg- licher bildgebenden Untersuchung stellt die Essenz der Informationsweitergabe dar. Vollständige und zweifelsfrei interpretierbare Ausgangsbefunde sind die Grundlage für Kontrolluntersuchungen und für den nachfolgenden Untersucher zur Bewertung etwaiger Veränderungen unabdingbar. Eine strukturierte Befunderhebung und Dokumentation ist im Rahmen der Fachartausbildung bisher kein Ausbildungsstandard, wird allerdings im Curriculum der DEGUMzertifizierten Ultraschallkurse adressiert und ist letztlich Qualitäts- und Zertifizierungsmerkmal der KV-Ultraschallvereinbarung. Letztere setzt die Empfehlungen und Vorgaben der DEGUM in der Ultraschallvereinbarung um.

Insbesondere unerfahrene Untersucher können von der Verwendung einer strukturierten Dokumentation profitieren, da während Erstellung des Befundberichtes auf relevante Inhalte sowie die Benennung anatomischer Strukturen hingewiesen und die empfohlene Terminologie angeboten wird [4, 5]. Leitenden Ärzten obliegt es, die Befunderstellung und die -qualität während der Facharztausbildung zu supervidieren.

Unsere Daten zeigen, dass die strukturierte Befunderhebung ggf. unter Verwendung von Textbausteinen mit einer Verbesserung der Dokumentationsvollständigkeit einhergeht (88,5\% vs. $75 \%)$. Die Ergebnisse lassen den zusätzlichen Nutzen von standardisierter digitaler Befunderhebung und Archivierung erkennen. Die Einführung und Umsetzung der dargestellten Maßnahmen sollten daher dringend angestrebt werden. Erkennbar wird, dass Handlungsbedarf besteht, denn nur $60 \%$ der an der Studie teilnehmenden Abteilungen dokumentieren digital, und nur 2 der mitwirkenden Kliniken verfügen über eine integrierte digitale Befund- und Bilddokumentation. An die erfolgreiche Einführung digitaler Lösungen besteht ein hoher Anspruch, denn sie müssen eine hohe und variable Schnittstellenkompatibilität aufweisen, den technischen Support garantieren, einfach, schnell und intuitiv in der Anwendung sein sowie die Anpassung durch den Benutzer ermöglichen. 


\section{Fazit für die Praxis}

Die Befundvollständigkeit und -qualität von routinemäßig angefertigten Kopf- und Halssonographiebefunden an einer Auswahl von HNO-Universitätskliniken ist insgesamt optimierbar. Die Implementierung strukturierter Befundmasken, die Umstellung der analogen Dokumentation auf digitale Lösungen sowie die Vernetzung in das KIS/PACS der Kliniken sollte weiter gefördert werden. Leitende Ärzte in Kliniken sind dazu angehalten, die Befundqualität unerfahrener Kollegen regelmäßig zu prüfen und auf die Einhaltung entsprechender Standards zu achten.

\section{Korrespondenzadresse}

\section{PD Dr. J. Künzel}

Klinik und Poliklinik für Hals-Nasen-OhrenHeilkunde, Universitätsklinikum Regensburg Franz-Josef-Strauß-Allee 11, 93053 Regensburg, Deutschland

julian.kuenzel@ukr.de

Funding. Open Access funding enabled and organized by Projekt DEAL.

\section{Einhaltung ethischer Richtlinien}

Interessenkonflikt. J. Künzel, A. Bozzato, B.P. Ernst T. Fuhrmann, I. Ugele, C. Scherl, M. Schapher, G.F. Volk, N. Mansour, A. Knopf, C. Bohr und K.-F. Hamann geben an, dass kein Interessenkonflikt besteht.

Für diesen Beitrag wurden von den Autoren keine Studien an Menschen oder Tieren durchgeführt. Für die aufgeführten Studien gelten die jeweils dort angegebenen ethischen Richtlinien.

Open Access. Dieser Artikel wird unter der Creative Commons Namensnennung 4.0 International Lizenz veröffentlicht, welche die Nutzung, Vervielfältigung, Bearbeitung, Verbreitung und Wiedergabe in jeglichem Medium und Format erlaubt, sofern Sie den/die ursprünglichen Autor(en) und die Quelle ordnungsgemäß nennen, einen Link zur Creative Commons Lizenz beifügen und angeben, ob Änderungen vorgenommen wurden.

Die in diesem Artikel enthaltenen Bilder und sonstiges Drittmaterial unterliegen ebenfalls der genannten Creative Commons Lizenz, sofern sich aus der Abbildungslegende nichts anderes ergibt. Sofern das betreffende Material nicht unter der genannten Creative Commons Lizenz steht und die betreffende Handlung nicht nach gesetzlichen Vorschriften erlaubt ist, ist für die oben aufgeführten Weiterverwendungen des Materials die Einwilligung des jeweiligen Rechteinhabers einzuholen.
Weitere Details zur Lizenz entnehmen Sie bitte der Lizenzinformation auf http://creativecommons.org/ licenses/by/4.0/deed.de.

\section{Literatur}

1. Armbruster M, Gassenmaier $S$, Haack $M$ et al (2018) Structured reporting in petrous bone MRI examinations: impact on report completeness and quality. Int J Comput Assist Radiol Surg 13:1971-1980

2. Beltz A, Gösswein D, Zimmer S et al (2018) Staging of oropharyngeal carcinomas: new TNM classification as a challenge for head and neck cancer centers. HNO66:375-382

3. Ernst BP, Hodeib M, Strieth S et al (2019) Structured reporting of head and neck ultrasound examinations. BMCMed Imaging 19:25

4. Ernst BP, Katzer F, Künzel J et al (2019) Impact of structured reporting on developing head and neck ultrasound skills. BMCMed Educ 19:102

5. Ernst BP, Strieth S, Katzer F et al (2020) The use of structured reporting of head and neck ultrasound ensures time-efficiency and report quality during residency. Eur Arch Otorhinolaryngol 277:269-276

6. European Society of Radiology (2018) ESR paper on structured reporting in radiology. Insights Imaging 9:1-7

7. Furukawa MK, Furukawa M (2010) Diagnosis of lymph node metastases of head and neck cancer and evaluation of effects of chemoradiotherapy using ultrasonography. Int JClin Oncol 15:23-32

8. Gassenmaier S, Armbruster M, Haasters F et al (2017) Structured reporting of MRI of the shoulder-improvement of report quality? Eur Radiol 27:4110-4119

9. Heyduck A, Jecker P, Bozzato A (2018) A germanwide inquiry about the significance of ultrasound in the head and neck area. Laryngorhinootologie 97:392-397

10. Kim SH, Sobez LM, Spiro JE et al (2020) Structured reporting has the potential to reduce reporting times of dual-energy $\mathrm{x}$-ray absorptiometry exams. BMC Musculoskelet Disord 21:248

11. Künzel J, Bozzato A, Strieth S (2017) Followup ultrasound of head and neck cancer. HNO 65:939-952

12. Künzel J, Strieth S, Wirth G et al (2018) Ultrasound in the re-staging of cervical metastases after chemoradiotherapy for head and neck cancer. Ultraschall Med 39:659-666

13. KVB (2020) Vereinbarung von Qualitätssicherungsmaßnahmen nach $\S 135$ Abs. 2 SGB V zur Ultraschalldiagnostik

14. Morgan TA, Helibrun ME, Kahn CE Jr (2014) Reporting initiative of the Radiological Society of North America: progress and new directions. Radiology 273:642-645

15. Nörenberg D, Sommer WH, Thasler $W$ et al (2017) Structured reporting of rectal magnetic resonance imaging in suspected primary rectal cancer: potential benefits for surgical planning and interdisciplinary communication. Invest Radiol 52:232-239

16. Psychogios G, Rueger $H$, Jering $M$ et al (2019) Ultrasound can help to indirectly predict contact of parotid tumors to the facial nerve, correct intraglandular localization, and appropriate surgical technique. Head Neck 41:3211-3218

17. Sabel BO, Plum JL, Kneidinger N et al (2017) Structured reporting of $\mathrm{CT}$ examinations in acute pulmonary embolism. J Cardiovasc Comput Tomogr 11:188-195

18. Schoeppe F, Sommer WH, Nörenberg D et al (2018) Structured reporting adds clinical value in primary CT staging of diffuse large B-cell lymphoma. Eur Radiol 28:3702-3709

19. Schöppe F, Sommer WH, Schmidutz F et al (2018) Structured reporting of $x$-rays for atraumatic shoulder pain: advantages over free text? BMC Med Imaging 18:20

20. Steinkamp HJ, Cornehl M, Hosten N et al (1995) Cervical lymphadenopathy: ratio of long- to shortaxis diameter as a predictor of malignancy. $\mathrm{Br} J$ Radiol 68:266-270

21. Tuncyurek O, Garces-Descovich A, JaramilloCardoso A et al (2019) Structured versus narrative reporting of pelvic MRI in perianal fistulizing disease: impact on clarity, completeness, and surgical planning. Abdom Radiol (NY) 44:811-820

22. Ying M, Ahuja A, Brook F et al (1996) Sonographic appearance and distribution of normal cervical lymph nodes in a Chinese population. JUltrasound Med 15:431-436 